\title{
Diabetes gestacional: seguimento após o parto
}

\section{Gestational diabetes: follow-up after delivery}

O conceito de diabetes melito gestacional (DMG) foi modificado nas últimas décadas em função da forma de se fazer o diagnóstico, o momento e os pontos de corte considerados. Isso ocorreu particularmente após a publicação dos resultados do estudo Hyperglycemia and Adverse Pregnancy Outcome (HAPO), que incluiu mais de 25.000 gestantes avaliadas para DMG. Desta forma, o número de gestantes consideradas portadoras de DMG teve um aumento significativo. Aos casos de diabetes diagnosticados na gestação, somam-se aqueles de diabetes melito pré-gestacional (tipos 1 ou 2), que correspondem a menos de $30 \%$ do total de diabéticas durante a gestação ${ }^{1,2}$.

O acompanhamento de gestantes com diabetes na gestação inclui o bom controle metabólico mediante o ajuste dos pilares terapêuticos: dieta, exercício físico e medicação, além do acompanhamento pré-natal realizado por uma equipe multiprofissional capacitada para este tipo de atendimento. Por recomendação da Organização Mundial de Saúde (OMS), a droga de escolha ao controle do metabolismo na gestação ainda é a insulina ${ }^{2}$.

Os critérios para definição do momento do parto já estão bem definidos, sendo o mesmo proposto para o termo, nas gestações bem controladas, sem macrossomia, malformações ou poli-hidrâmnio, além da ausência de comorbidades como hipertensão arterial. O momento do parto pode, eventualmente, ser antecipado para idades mais precoces nos casos com complicações fetais ou obstétricas.

Independentemente da via de parto e idade gestacional por ocasião do mesmo, é muito importante o seguimento puerperal dessas gestantes. Um dos aspectos mais negligenciados nesse período é a prescrição nos dias que se seguem ao parto, logo a dieta deve ser mantida, a ingesta hídrica estimulada e, a respeito da medicação, várias alternativas são possíveis. Dependendo do tipo de diabetes presente, pode-se optar:

- para os casos de diabetes diagnosticado durante a gestação, recomenda-se a suspensão das medicações para controle de diabetes, mantendo-se a dieta e incentivando deambulação precoce e amamentação exclusiva e à livre demanda;

- para as pacientes com diabetes anterior à gestação do tipo 2 que usavam insulina, deve-se reduzir a dose da mesma à quantidade usada antes da gestação e orientar a puérpera a comparecer à Unidade Básica de Saúde ou ao seu médico clínico para ajuste de sua medicação em cerca de seis semanas;

Correspondência

Belmiro Gonçalves Pereira Rua Alexander Fleming, 101 CEP: 13083-881

Campinas (SP), Brasil

Recebido

$22 / 07 / 2014$

Aceito com modificaçōes

$23 / 09 / 2014$

'Departamento de Ginecologia e Obstetrícia, Faculdade de Ciências Médicas, Universidade Estadual de Campinas - UNICAMP Campinas (SP), Brasil.

Conflito de interesses: não há. 
- para as diabéticas com tipo 1 , a recomendação é o retorno à dose pré-concepcional ou, em certos casos, um terço da dose de final da gestação ${ }^{3}$.

A avaliação e a prescrição no primeiro dia após o parto incluem atenção redobrada à involução uterina, aos cuidados especiais com as feridas cirúrgicas e com as mamas. Para a prescrição, é necessária a observação da necessidade de antibióticos, analgésicos e anti-inflamatórios. Deve-se lembrar que, no caso de parto normal sem lesões do canal de parto e sem episiotomia, não é necessária a prescrição de anti-inflamatórios, nem de antibióticos e só se deve prescrever analgésico em caso de necessidade. Nas puérperas de parto operatório, além do analgésico, deve-se prescrever anti-inflamatórios e, nos casos de cesárea, antibiótico profilático.

Em relação ao diabetes gestacional, é importante recordar que esse é um indicador preditivo da possibilidade do desenvolvimento de diabetes no futuro, o que implica na orientação da mulher quanto à necessidade de investigação periódica para tal enfermidade. $\mathrm{O}$ fato de ter tido diabetes gestacional em uma gestação coloca a mulher também em condição de altíssimo risco para o desenvolvimento de diabetes em gestações futuras, o que implica no preparo da mesma para tais gestações. Cerca de um terço das gestantes portadoras de DMG desenvolverá diabetes ou intolerância à glicose no seguimento pós-parto. Para tanto, é necessária a realização periódica de testes para investigação de intolerância à glicose ou mesmo de diabetes ${ }^{2,3}$.

Os critérios para diagnóstico de diabetes no período puerperal remoto estão estabelecidos e devem ser pesquisados. Conforme recomendações da American Diabetes Association e da World Health Organization (WHO), devem ser considerados: porcentagem de hemoglobina glicada $\left(\mathrm{HbA}_{1 \mathrm{c}}\right)<6,5 \%$; glicemia de jejum de pelo menos 8 horas $\geq 126 \mathrm{mg} / \mathrm{dL}(7,0 \mathrm{mmol} / \mathrm{L})$; glicemia pós-sobrecarga de $75 \mathrm{~g}$ de glicose anidra dissolvida em água $\geq 200 \mathrm{mg} / \mathrm{dL}(11,1 \mathrm{mmol} / \mathrm{L})$ ou, em pacientes com sintomas de hiperglicemia, a dosagem ao acaso da glicemia $\geq 200 \mathrm{mg} / \mathrm{dL}(11,1 \mathrm{mmol} / \mathrm{L})^{2,3}$.

Para se proceder tal avaliação, é necessária a disponibilização de métodos contraceptivos eficazes, bem tolerados e que permitam planejar as próximas gestações. Desta forma, a revisão pós-parto feita na unidade em que foi realizado o acompanhamento do pré-natal pode ser uma boa recomendação, visto que a equipe deverá estar apta ao diagnóstico e ao preparo da mulher para a concepção. Seguramente, o controle de comorbidades, como hipertensão arterial, obesidade e outras síndromes metabólicas, pode contribuir para um melhor planejamento da gestação futura, assim como da sua evolução mais tranquila e com melhores resultados como, por exemplo, a redução da probabilidade de desenvolver diabetes em uma futura gestação. Alguns exames de laboratório podem ser úteis nessa fase: a dosagem da glicemia de jejum que deve estar inferior a $100 \mathrm{mg} / \mathrm{dL}$, glicemia ao acaso inferior a $200 \mathrm{mg} / \mathrm{dL}$ ou a porcentagem de $\mathrm{HbA}_{1 \mathrm{c}}$ inferior a 6,5\%. Nessas situações, é dispensável a realização de curva glicêmica com sobrecarga de glicose. $\mathrm{Na}$ eventualidade da necessidade de realização do teste de sobrecarga, segue-se o padrão sugerido pela OMS com uma dosagem 2 horas após uma sobrecarga de 75 g de glicose anidra, a qual deverá ser inferior a $200 \mathrm{mg} / \mathrm{dL}^{2,4}$.

O estabelecimento de metas nessa fase é muito útil. A redução do peso, por exemplo, pode ser medida recomendada e, de modo geral, bem aceita pela mulher, estimulada pelo resultado da gestação e pelas dificuldades por ela enfrentadas para alcançá-la. A prática de exercícios físicos como mudança no estilo de vida também é relativamente bem aceita e deve ser fortemente estimulada e incentivada. Raramente, necessita-se de medicação para essas pacientes.

É sabido que o período puerperal nas mulheres lactantes em amamentação exclusiva e à livre demanda garante um período variável de infertilidade. Contudo, o retorno à fertilidade pode ser melhor controlado quando se associam métodos de contracepção eficazes, que não interfiram com a lactação nem com o controle do metabolismo glicêmico. Portanto, a prescrição de método contraceptivo adequado e desejado pala cliente pode ser importante. Para tanto, é necessário o conhecimento dos métodos e sua real utilidade e eficácia neste período, assim como possíveis efeitos indesejáveis 5 .

Para acertar na escolha do método, é necessário lembrar que apenas a eficácia contraceptiva não basta. Deve-se certificar-se de que o método não interfira com a lactação, com o peso da paciente, com a pressão arterial ou com doenças vasculares eventualmente presentes ou associadas com o distúrbio do metabolismo. Entre as opções de métodos contraceptivos, destacam-se: pílulas de progestógeno (para uso contínuo), que modificam as características do muco cervical; pílulas combinadas, que, por conterem estrogênio, não são recomendadas na fase de lactação; hormonais injetáveis, como o mensal que, também, por conter estrogênio não se usa durante a amamentação; injetável trimestral com acetato de medroxiprogesterona na dose de $150 \mathrm{mg}$ a cada três meses, que não interfere com a lactação, porém, pode interferir com a tão desejada redução de peso. Desta forma, embora muitos estejam disponíveis e possam ser utilizados sem maiores riscos, o dispositivo intrauterino (DIU) pode ser a melhor escolha para o controle da fertilidade neste grupo específico de mulheres. O DIU com levonorgestrel de liberação lenta pode ter indicação muito precisa para o período 
puerperal de mulheres que tiveram diabetes gestacional e têm planos de programar novas gestações ${ }^{6,7}$.

\section{Conclusões}

O período pós-parto nas mulheres portadoras de diabetes diagnosticado na gestação ou com hiperglicemia gestacional é um importante momento para se estimular a amamentação, orientar o planejamento de futuras gestações e fazer prevenção primária do diabetes melito mediante medidas que visem o controle de obesidade. Nos casos em que o risco para diabetes decorre do fato das mulheres terem sido portadoras de DMG, deve-se fazer a prevenção secundária com prescrição de dieta, exercício físico e, eventualmente, medicação. Para tanto, conhecimento dos métodos contraceptivos úteis e não prejudiciais ao controle do metabolismo e peso maternos é fundamental.

\section{Referências}

1. Metzger BE, Lowe LP, Dyer AR, Trimble ER, Chaovarindr U, Coustan DR, et al. Hyperglycemia and adverse pregnancy outcomes. N Engl J Med. 2008;358(19):1991-2002.

2. World Health Organization. Diagnostic criteria and classification of hyperglycaemia first detected in pregnancy. Geneva: WHO; 2013.

3. American Diabetes Association. Diagnosis and classification of diabetes mellitus. Diabetes Care. 2014;37(Suppl 1):S81-90.

4. Committee on Practice Bulletins-Obstetrics. Practice Bulletin n. 137: gestational diabetes mellitus. Obstet Gynecol. 2013;122(2 Pt 1):406-16.
5. Kitzmiller JL, Dang-Kilduff L, Taslimi MM. Gestational diabetes after delivery: short-term management and long-term risks. Diabetes Care. 2007;30(Suppl 2):S225-35.

6. Kim C. Managing women with gestational diabetes mellitus in the postnatal period. Diabetes Obes Metab. 2010;12(1):20-5.

7. Damm P, Matthiesen ER, Petersen KR, Kjos S. Contraception after gestational diabetes. Diabetes Care. 2007;30/Suppl 2):S236-41. 